\title{
Epidemiology of HPV genotypes in Uganda and the role of the current preventive vaccines: A systematic review
}

Cecily Banura ${ }^{1 *}$, Florence M Mirembe ${ }^{2}$, Anne R Katahoire ${ }^{1}$, Proscovia B Namujju ${ }^{3,4}$, Anthony K Mbonye ${ }^{5}$ and Fred M Wabwire ${ }^{6}$

\begin{abstract}
Background: Limited data are available on the distribution of human papillomavirus (HPV) genotypes in the general population and in invasive cervical cancer (ICC) in Uganda. Yet, with the advent of preventive HPV vaccines that target HPV 16 and 18 responsible for causing about 70\% of ICC cases in the world, such information is crucial to predict how vaccination and HPV-based screening will influence prevention of ICC.

Methods: To review the distribution of HPV infection and prevalent genotypes, electronic databases (e.g. PubMed/ MEDLINE and HINARI) were searched for peer reviewed English articles on HPV infection up to November 30, 2010. Eligible studies were selected according to the following criteria: DNA-confirmed cervical or male genital HPV prevalence and genotypes, HPV incidence estimates and HPV seroprevalence among participants.

Results: Twenty studies were included in the review. Among HIV negative adult women, the prevalence of HR-HPV infections ranged from 10.2\% -40.0\% compared to 37.0\%-100.0\% among HIV positive women. Among HIV positive young women aged below 25 years, the prevalence of HR-HPV genotypes ranged from $41.6 \%-75.0 \%$ compared to 23.7\% -67.1\% among HIV negative women. Multiple infections with non vaccine HR-HPV genotypes were frequent in both HIV positive and HIV negative women. The main risk factors for prevalent HPV infections were age, lifetime number of sexual partners and HIV infection. Incident infections with HR-HPV genotypes were more frequent among adult HIV positive than HIV negative women estimated at 17.3 and 7.0 per 100 person-years, respectively. Similarly, incident HR-HPV among young women aged below 25 years were more frequent among HIV positive (40.0 per 100 person-years) than HIV negative women (20.3 per 100 person-years) women. The main risk factor for incident infection was HIV infection. HPV 16 and 18 were the most common genotypes in ICC with HPV 16/18 contributing up to $73.5 \%$ of cases with single infections.

Among uncircumcised adult HIV positive males, HR-HPV prevalence ranged from 55.3\% -76.6\% compared to 38.6\% $-47.6 \%$ in HIV negative males. Incident and multiple HR-HPV infections were frequent in HIV positive males. Being uncircumcised was the main risk factor for both prevalent and incident HPV infection.
\end{abstract}

Conclusion: Infections with HR-HPV genotypes were very common particularly among HIV positive individuals and young women irrespective of HIV status. Given the high prevalence of HIV infection, HPV-associated conditions represent a major public health burden in Uganda. However, although the most common HPV genotypes in ICC cases in Uganda were those targeted by current preventive vaccines, there were a large number of individuals infected with other HR-HPV genotypes. Technology allowing, these other HR-HPV types should be considered in the development of the next generation of vaccines.

\footnotetext{
* Correspondence: cbanura@chdc.mak.ac.ug

${ }^{1}$ Child Health and Development Centre, Makerere University College of

Health Sciences, P. O. Box 6717, Kampala, Uganda

Full list of author information is available at the end of the article
} 


\section{Introduction}

Invasive cervical cancer (ICC) remains a leading cause of death among women in low-resource settings [1] and virtually every case of ICC is caused by HPV [2]. Genital HPV infection is predominantly, but not exclusively, a sexually transmitted infection and about $50 \%$ to $80 \%$ sexually active women are infected by this virus at least once in their lifetime [3,4]. Worldwide, women in developing countries account for about $85 \%$ of both annual cases of ICC estimated at 493,000 and annual deaths from ICC estimated at 273,500 [1]. Men play an important role in HPV transmission as HPV DNA has been detected in the genitalia of up to $73 \%$ of healthy men [5].

Over $100 \mathrm{HPV}$ genotypes have been identified and are divided into high-risk (HR) and low risk (LR) depending on their potential to cause cancer. There are about 15 cancer causing HPV genotypes that are responsible for $5 \%$ of all human cancers but predominantly cervical cancer and other anogenital cancers [6]. HPV 16 and HPV 18 are the most virulent HR-HPV genotypes causing about $70 \%$ of all ICC in the world [7]. Two preventive vaccines targeting HPV 16 and 18 have been developed and are currently licensed in over 100 countries including Uganda; Cervarix ${ }^{\circledR}$, a bivalent HPV 16/18 from GlaxoSmithKline Biologicals (GSK) and Gardasil ${ }^{\mathbb{}}$, a quadrivalent HPV 6/11/16/18 vaccine from MSD Merck. Gardasil also targets two LR-HPV genotypes 6 and 11 , which together cause $90 \%$ of genital warts [8]. These current preventive HPV vaccines however, offer protection only against a few cancer causing HPV genotypes.

Uganda has a large burden of ICC. Current estimates indicate that every year, 3,577 women are diagnosed with ICC and 2,464 die from the disease. Compared to the USA, the age adjusted cervical cancer incidence per 100,000 population per year is 8.3 times higher in Uganda (47.5 vs.5.7) and age adjusted death rate as a result of ICC is 20.5 times higher (34.9 vs. 1.7) [9]. The high incidence of ICC is a reflection of prevalent HRHPV infections and failure to prevent their clinical manifestations by effective screening programs that have dramatically reduced incidence in developed countries [10]. The introduction of Cervarix ${ }^{\circledR}$ by the Program for Appropriate Technology for Health (PATH) in partnership with the Uganda Ministry of Health may offer the greatest hope of significant reduction of ICC burden provided the vaccine is widely and properly delivered [11]. Furthermore, harmonizing primary (vaccination) and secondary prevention (screening) will result in greater reduction of ICC than either program alone [12].

Limited data are however, available on the distribution of HPV genotypes in the general population and in ICC in Uganda. Yet, with the advent of HPV vaccination, such information is crucial for policy makers to predict how HPV vaccination and HPV-based screening will influence prevention of ICC. Moreover, even with a successful vaccination program, vaccinated women will still require screening to detect those that will develop ICC from other HR-HPV genotypes that are not prevented by current vaccines. Therefore, the objective of this review was to summarize published data on HPV infection in Uganda. This review will provide useful baseline information for evaluating the impact of preventive HPV vaccines and HPV based technologies used in screening programs.

\section{Methods}

PubMed/MEDLINE and HINARI websites were searched for peer reviewed English language published medical literature on HPV infection in Uganda up to November 30, 2010. The search was conducted using the following medical subject heading (MESH) and text words either singly or in combination: "HPV", "genotypes"," cervical cancer", "screening", "vaccination", "serology" and "Uganda". Eligible studies were selected according to the following criteria: DNA-confirmed cervical or male genital HPV prevalence, HPV incidence estimates and HPV seroprevalence among participants. From each article, information on first author, publication journal and date, dates of sample collection, HPV detection methods, study design, anatomical site and methods of sample collection, population description, mean or median age of sample with range when available, sample size, the prevalence of HIV when available, the prevalence of HPV (overall, high risk, low risk, specific genotypes) as well as the prevalence of HPV among HIV positive and HIV negative women. Risk factors for HPV infection were also extracted when available.

Twenty studies were found and were included in the review: 9 prevalence studies, 2 prospective cohort studies in women, 3 randomized controlled trials in men, 4 studies on ICC, and 2 seroprevalence studies.

\section{Methods used for the detection of HPV DNA, typing for specific genotypes and to determine seroprevalence}

Various techniques and assays were used for detection of HPV DNA and genotyping. Polymerase Chain Reaction $^{\mathrm{TM}}$ (PCR) using generic or consensus primers and Hybrid capture ${ }^{\mathrm{TM}} 2$ (HC2, Digene Co., Gaithersburg, MD, USA) were used in majority of studies. Both techniques have been optimized to detect 13 HR-HPV genotypes [13]. However, some studies used the Southern Blot technique which generally has low sensitivity and detects only a few HR-HPV genotypes. One HPV seroprevalence study was done before the World Health Organization (WHO) developed international standard reagents for calibration of HPV DNA assays and kits 
[14]. Therefore, caution should be taken when interpreting results from the different studies as the sensitivities and specificities of the HPV assays vary widely depending on the type and quality of the biological specimen and the type and quality of reagents used. Additionally, assays differ in their ability to detect different HPV genotypes particularly where there are co-infections with multiple HPV types [15].

\section{Results}

The findings from studies conducted in selected populations across the country are summarized in Tables 1,2 , $3,4,5,6$.

Prevalence of HPV infections in population-and clinicbased studies of women with and without cervical abnormalities

In women below 25 years, $71.8 \%$ of women who were positive for HPV 16 and 18 were also infected with other HR-HPV genotypes [16]. Compared to HIV negative women, HIV positive women had more HR-HPV genotypes detected in cervical/vaginal specimens [16-19] and HSIL lesions [17]. The mean number of HPV genotypes detected was higher among HIV positive compared to HIV negative women (2.8; range 1-9) and 2.1; range $1-10$, respectively; [ $t$-test, $3.88 ; \mathrm{p}<0.001$ ]) [16]. Likewise, among young primiparous women, the mean number of HPV types detected among HIV positive women was higher (2.4, range 1-10) than among HIV negative women $(1.8$, range $1-12)$ [Student's t-test $=$ 2.79, $\mathrm{p}=0.005$ ] [18]. HIV positive women were four times more likely to have abnormal cytology than HIV negative women (43\% vs. 11.6\%, p < 0.001) [19]. HPV positive women co-infected with HIV had other HRHPV genotypes that did not include HPV 16 or 18 [16,17].

\section{Risk factors for prevalent HPV infection}

Few studies evaluated risk factors for HPV infections. Lifetime number of sexual partners [16,20]; HIV positivity $[16,21]$; young age at first sexual intercourse [16] and young age $[16,21]$ were significantly associated with HPV infection. Relative to women in 19-25 age-group, the odds of detecting HR-HPV decreased by $48 \%, 60 \%$, and $79 \%$ among women in 26-30, 31-37, and 38-49 agegroup [21]. On average, the odds of detecting HR-HPV decreased by $9 \%$ for each-year increase in age. However, HPV was only inversely associated with age $\left(\mathrm{P}_{\text {trend }}\right.$ 0.001 ) in HIV negative women as the prevalence of HPV among HIV positive women seemed to remain high in all age-groups [20]. Other risk factors found included: abnormal cytology $[17,18]$; employment in a tertiary sector, concurrent pregnancy, presence of genital warts, and testing positive for Syphilis, Chlamydia trachomatis and Neisseria Gonnorhea [16]. Smoking cigarettes the previous year was significantly associated with HPV infection after controlling for age, age at sexual debut and HIV status (Adjusted OR = 3.74; 95\% CI: 1.15-12.16) [21].

HPV 16 and 18 were the most frequent genotypes in ICC cases. Furthermore, results of pooled data compiled by the International Agency for Research on Cancer (IARC) showed a prevalence of HPV 16/18 of $74.1 \%$ (95\% CI: 66.4-80.8) [22,23].

\section{Incident and risk factors for HPV infections among women}

HIV positive women were twice as likely to have incident infection compared to HIV negative women [24]. Risk factors for incident HPV infections were HIV positivity $[24,25]$; young age, many lifetime and recent sexual partners and single women [24,25].

\section{Risk factor for HPV infection in males participating in circumcision trials}

Not being circumcised was the main risk factor for both prevalence and incident HR-HPV infections.

\section{HPV serology}

HPV seroprevalence peaked in women below 20 years of age. The most common HR-HPV serotypes was HPV 33 (23\%), HPV 16 (21\%), HPV 31 (16\%), HPV 45 (11\%) and HPV 18 (8\%). The seroprevalence of LR-HPV 6, 11 and HPV 6/11 combined was $30 \%, 26 \%$, and $44 \%$, respectively. Of the 1,118 HPV seropositive women, 53\% had antibodies to more than one HPV genotype, 30\% had $2 \mathrm{HPV}$ genotypes, 14\% had $3 \mathrm{HPV}$ genotypes and 9\% had 4-7 genotypes [26]. Antibodies against HPV 16 were significantly associated with ICC (OR $=2.0 ; 95 \%$ CI: 1.2-3.1, p < 0.01) [27]. HPV seoprevalence was higher among HIV positive compared to HIV negative women, $74.0 \%$ and $56 \%$, respectively. Multiple antibody positivity was more frequent in HIV positive (53\%) than HIV negative women (28\%) [26].

\section{Discussion}

To the best of our knowledge, this systematic review represents the first ever to evaluate the burden of HPV infections and ICC in Uganda. It is worth noting that comparison of HPV prevalence and incidence rates across studies was hampered by differences in populations studied, laboratory methods used, and variation in HPV genotypes detected. Nevertheless, consistent with other studies in sub Saharan Africa [28], the review demonstrated a high burden of HR-HPV genotypes in the general population and in ICC. HPV 16 and 18 were the most common genotypes in ICC similar to the global HPV distribution pattern [29]. Mathematical models 
Table 1 HPV prevalence and genotype distribution in women with and without cervical abnormalities

\begin{tabular}{|c|c|c|c|c|c|c|}
\hline Author & Area, subjects & $\begin{array}{l}\text { Study } \\
\text { design }\end{array}$ & HPV test & $\begin{array}{l}\text { HPV prevalence for any, } \\
\mathrm{HR}^{*}, \mathrm{LR}^{* *} \text { and specific } \\
\text { genotypes among all } \\
\text { tested women or only } \\
\text { HPV positive women }\end{array}$ & 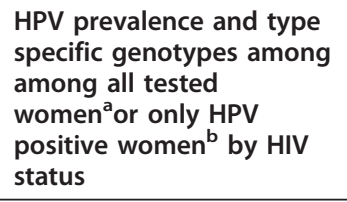 & Comments \\
\hline \multicolumn{7}{|c|}{ Population-Based studies } \\
\hline $\begin{array}{l}\text { Serwadda } \\
\text { et al., } 1999 \\
\text { [52] }\end{array}$ & $\begin{array}{l}\text { Rakai district, Random sample } \\
\text { of } 960 \text { women aged } 15-59 \\
\text { years using self collected } \\
\text { vaginal swabs }\end{array}$ & $\begin{array}{l}\text { Cross- } \\
\text { sectional }\end{array}$ & $\begin{array}{l}\text { Hybrid } \\
\text { Capture } 2\end{array}$ & Any HR-HPV ${ }^{a}, 16.7 \%$ & $\begin{array}{l}\text { HIV status }^{a} \\
\text { HIV+, } 44.3 \% \\
\text { HIV-, } 10.2 \%\end{array}$ & $\begin{array}{l}\text { HIV } \\
\text { prevalence }{ }^{a}, \\
17.8 \%\end{array}$ \\
\hline $\begin{array}{l}\text { Safaeian } \\
\text { et al., } 2007 \\
{[53]}\end{array}$ & $\begin{array}{l}\text { Rakai district, } 606 \text { women, } \\
\text { median age } 30 \text { years (25-38 } \\
\text { years) }\end{array}$ & $\begin{array}{l}\text { Baseline of } \\
\text { a } \\
\text { population- } \\
\text { based } \\
\text { cohort } \\
\text { study }\end{array}$ & $\begin{array}{l}\text { Hybrid } \\
\text { Capture 2/ } \\
\text { PGMY 09/11 } \\
\text { assay }\end{array}$ & $\begin{array}{l}\text { Any HR-HPV }{ }^{a}, 19 \% \\
\text { Type-specific genotypes } \\
\text { HPV 16, } 4.5 \% \\
\text { HPV 52, } 4.4 \% \\
\text { HPV 66, 3.0\% } \\
\text { HPV 58, 3.0\% }\end{array}$ & $\begin{array}{l}\text { HIV status }^{a} \\
\text { HIV positive } \\
\text { Self-collected, 40\% } \\
\text { Physician collected, 37\% } \\
\text { HIV negative } \\
\text { Self-collected, 15\% } \\
\text { Physician collected, 16\% }\end{array}$ & $\begin{array}{l}\text { HIV } \\
\text { prevalence }{ }^{a}, \\
15.7 \%\end{array}$ \\
\hline $\begin{array}{l}\text { Asiimwe } \\
\text { et al., } 2008 \\
\text { [21] }\end{array}$ & $\begin{array}{l}\text { Bushenyi district, } 314 \text { women } \\
\text { with median age of } 27 \text { years } \\
\text { (range,18-49) using self } \\
\text { collected vaginal swabs }\end{array}$ & $\begin{array}{l}\text { Cross- } \\
\text { sectional }\end{array}$ & $\begin{array}{l}\text { Hybrid } \\
\text { Capture } 2 \\
\text { assay }\end{array}$ & Any HR-HPV', $17.2 \%$ & & $\begin{array}{l}\text { HIV } \\
\text { prevalence } \\
5.6 \% \text { (self- } \\
\text { reported) }\end{array}$ \\
\hline $\begin{array}{l}\text { Safaeian } \\
\text { et al., } \\
\text { 2008b [20] }\end{array}$ & $\begin{array}{l}\text { Rakai district, } 926 \text { sexually } \\
\text { exprienced women aged } 15-49 \\
\text { years, mean age } 26 \text { years, who } \\
\text { provided at least } 3 \text { consecutive } \\
\text { self-collected swabs }\end{array}$ & $\begin{array}{l}\text { Population- } \\
\text { based } \\
\text { cross- } \\
\text { sectional } \\
\text { study }\end{array}$ & $\begin{array}{l}\text { Hybrid } \\
\text { Capture 2/ } \\
\text { Prototype } \\
\text { Roche } \\
\text { reverse line } \\
\text { blot assay }\end{array}$ & $\begin{array}{l}\text { Any HR-HPV }{ }^{a}, 19.2 \% \\
\text { Type-specific genotypes } \\
\text { HPV 52, } 2.2 \% \\
\text { HPV } 16,2.1 \% \\
\text { HPV 51, } 1.7 \% \\
\text { HPV } 66,1.5 \% \\
\text { HPV } 68,1.3 \%\end{array}$ & $\begin{array}{l}\text { HIV status }^{a} \\
\text { HIV+, } 46.6 \% \\
\text { HIV-, } 14.8 \%\end{array}$ & $\begin{array}{l}\text { HIV } \\
\text { prevalence } \\
13.7 \%\end{array}$ \\
\hline
\end{tabular}

\section{Clinic-Based Studies}

Adult women

\author{
Blossom \\ STI clinic in Kampala, 106 \\ women mean age 26.3 years \\ [17] \\ (range,18-51)
}

Cross-

sectional

Roche PCR/
reverse strip
assay

Any HPV ${ }^{\mathrm{a}}, 46.2 \%$

Type-specific genotypes ${ }^{b}$

HPV 16/18, 18.4\%

HPV 52, $14.2 \%$

HPV $16,7.5 \%$

HPV 58, 7.5\%

Young women below 25 years

Banura Clinic for teenagers in Kampala,

et al., $\quad 1275$ sexually active young

2008a [16] women median age 20 years (range 12-24)
Baseline of $\mathrm{SPF}_{10} / \mathrm{LIPA} \quad$ Any HPV ${ }^{\mathrm{a}}, 74.6 \%$ a cohort PCR assay study
HR-HPV types ${ }^{\mathrm{a}}$, 51.4\%

Type-specific genotypes ${ }^{a}$ HPV 52, $13.2 \%$ HPV 51, $12.3 \%$ HPV 18, 10.7\% HPV 16, 10.6\% LR-HPV types ${ }^{\mathrm{a}}, 39.8 \%$ LR-HPV genotypes ${ }^{a}$ HPV 6, 15.5\% HPV 11, 13.3\%
Any HPV type ${ }^{a}$
HIV+, 59.5\%
HIV-, 39.1\%
HR-HPV types
HIV+, 100.0\%
HIV-, 88.9\%

HIV positive women Any HPV ${ }^{a}, 87.8 \%$

HR-HPV types, $67.1 \%$

Type-specific genotypes ${ }^{a}$

HPV 16, 18.3\%

HPV 18, 9.8\%

HPV 6, 15.9\%

HPV $11,17.1 \%$

HIV negative women

Any HPV ${ }^{a}, 73.2 \%$

HR-HPV types ${ }^{\mathrm{a}}, 49.7 \%$

Type-specific genotypes ${ }^{a}$

HPV 16, 10.7\%

HPV $18,12.1 \%$

HPV 6, 15.3\%

HPV 11, 12.7\%

HIV positive women

Any HPV ${ }^{a}, 72.2 \%$

Type-specific genotypes ${ }^{b}$

HPV 16, 18.1\%

HPV $18,6.9 \%$

HPV $6,8.3 \%$

HPV $11,1.4 \%$

HIV negative women

Any HPV ${ }^{\mathrm{a}}, 58.9 \%$

Type-specific genotypes ${ }^{b}$

HPV $16,7.7 \%$

HPV $18,5.7 \%$

HPV 6, 5.3\%

HPV $11,3.4 \%$
HIV

prevalence ${ }^{a}$, $34.9 \%$

HIV

prevalence $^{a}$, $8.6 \%$

HIV

prevalence ${ }^{\mathrm{a}}$, $7.3 \%$ 


\begin{tabular}{|c|c|c|c|c|}
\hline \multicolumn{5}{|c|}{ Screened adult women } \\
\hline $\begin{array}{l}\text { Buonaguro } \\
\text { et al., } 2000 \\
{[54]}\end{array}$ & $\begin{array}{l}\text { Nsambya hospital, Scrapes of } 16 \\
\text { women with normal ecto- } \\
\text { cervical epithelium and different } \\
\text { degrees of cervical epithelial } \\
\text { lesions }\end{array}$ & $\begin{array}{l}\text { Cross- } \\
\text { sectional }\end{array}$ & $\begin{array}{l}\text { Southern } \\
\text { Blot analysis }\end{array}$ & HPV $16^{a}, 12.5 \%$ \\
\hline $\begin{array}{l}\text { Taube } \\
\text { et al., } 2010 \\
{[19]}\end{array}$ & $\begin{array}{l}\text { Post-natal clinic at Mulago } \\
\text { hospital, Kampala } \\
\text { Cervical exfoliated cells from } \\
196 \text { women, } 4-12 \text { weeks post- } \\
\text { partum and aged between } 18 \text { - } \\
30 \text { years (mean age for HIV - } 23 \\
\pm 3 \text {; HIV }+25.4 \pm 3.2 \text { ) }\end{array}$ & $\begin{array}{l}\text { Cross- } \\
\text { sectional }\end{array}$ & $\begin{array}{l}\text { Roche Linear } \\
\text { array assay }\end{array}$ & $\begin{array}{l}\text { Any HR-HPV }{ }^{a}, 49.0 \% \\
\text { HPV 16/18 }{ }^{a}, 24 \% \\
\text { HR-HPV types } \\
\text { HPV } 16,8.2 \% \\
\text { HPV } 18,3.5 \% \\
\text { HPV } 45,5.1 \% \\
\text { HPV 58, 5.1\% } \\
\text { LR-HPV types } \\
\text { HPV 6, 2.6\% } \\
\text { HPV } 11,1.0 \%\end{array}$ \\
\hline
\end{tabular}

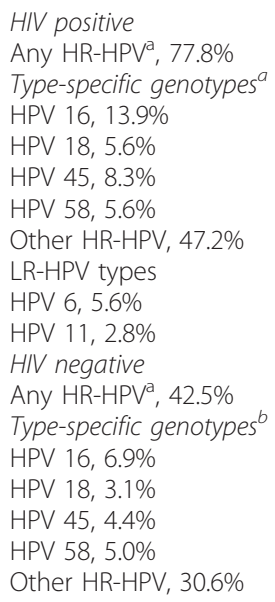

${ }^{*} \mathrm{HR}=$ High Risk; ${ }^{* *} \mathrm{LR}=$ Low Risk

a Denominator consist of all the tested women

${ }^{b}$ Denominator consist of only HPV positive women

predict that widespread use of preventive HPV vaccines containing genotypes $16 / 18$ have the potential to reduce deaths from ICC by $50 \%$ over several decades [30-32]. However, the efficacy of these vaccines in countries like Uganda with high prevalence of HIV infection, endemic malnutrition, malaria infection and intestinal worm infestation is not yet known. Our review also found that the third most frequent HPV genotype after HPV 16 and 18 among women with ICC was HPV 45, which differed from the worldwide distribution (HPV 16, 18, 58) [9]. Variation in HPV genotype distribution in ICC is not new as it has been observed in other regions of the world. It is hypothesized that host immunogenetic factors and biologic interplay between different HPV genotypes or variants are probably responsible [32]. This variation however, implies that any development of the next generation of multivalent preventive HPV vaccines should contain more HR-HPV genotypes than only 16/ 18. Based on data contained in this review, multivalent vaccines containing HPV 16/18/45 would potentially prevent approximately $83.1 \%$ of ICC in Uganda.

The highest prevalence and incidence rates of HPV infections occurred in young women below 25 years similar to previous studies [23]. It is important to note that Ugandan women tend to marry and become sexually active at a young age and often have older and more sexually experienced partners, which factors would put them at greater risk for HPV infection. The main risk factors for prevalent and incident HPV infections were age, number of lifetime number of sexual partners and HIV infection consistent with other studies [23,33].

$\mathrm{HIV}$ positive individuals had a high burden of HPV infection at the time when their life-spans are being prolonged by expanded access to highly active anti-retroviral therapy (HAART) and medical care. Since 2003, the HIV prevalence in Uganda has stabilized at about $6.4 \%$ among adults and by the end of 2010; approximately 1.1 million were living with HIV [34]. Women were disproportionately affected accounting for about $57 \%$ of the total adults living with HIV [34]. However, the proportion of co-infection with HIV and HPV is not known. Though several studies have consistently shown a high burden of HPV-associated diseases in HIV positive women even in the era of HAART [35], presently, there is limited or noexistent routine screening services for many HIV positive women. There are advantages to providing routine screening to HIV positive women. In Zambia, for example, routine screening prevented one death from ICC for every 46 HIV positive women screened [36]. Presently, there is limited data to support or discourage HPV vaccination of HIV positive individuals. To date, immune response to HPV vaccination among HIV positive individuals using the quadrivalent vaccine is limited to a small study of 120 children aged 7-11 years some of whom used anti retroviral therapy, which was conducted in the USA [37]. The antibodies developed by $>99.5 \%$ of the vaccinated children were much lower than in HIV negative historical controls 
Table 2 HPV incidence in women without cervical abnormalities

\begin{tabular}{|c|c|c|c|c|c|c|}
\hline Author & Area, subjects & $\begin{array}{l}\text { Study } \\
\text { design }\end{array}$ & $\begin{array}{l}\text { HPV } \\
\text { test }\end{array}$ & $\begin{array}{l}\text { HPV incidence rate for any, } \mathrm{HR}^{*} \text {, } \\
\mathrm{LR}^{* *} \text { and type-specific } \\
\text { genotypes among all tested } \\
\text { women }^{\mathrm{a}} \text { per } 100 \mathrm{PYs}\end{array}$ & $\begin{array}{l}\text { HPV incidence for any and } \\
\text { type-specific genotypes all } \\
\text { tested women }{ }^{\text {a }} \text { per } 100 \text { PYs } \\
\text { by HIV status }\end{array}$ & Comments \\
\hline $\begin{array}{l}\text { Safaeian } \\
\text { et al., } \\
2008 \mathrm{a} \\
{[24]}\end{array}$ & $\begin{array}{l}\text { Rakai district, rural women, } \\
\text { median age } 29 \text { years (range, } \\
15-59 \text { years) }\end{array}$ & $\begin{array}{l}\text { Prospective } \\
\text { cohort }\end{array}$ & $\begin{array}{l}\text { HC 2/ } \\
\text { PGMY/ } \\
09 / 11 \\
\text { assay }\end{array}$ & $\begin{array}{l}\text { Any HR-HPV }{ }^{a}, 8.7 \\
\text { Type-specific genotypes } \\
\text { HPV } 16,1.5 \\
\text { HPV } 18,0.9 \\
\text { HPV 45, } 1.1 \\
\text { HPV 51, } 1.8 \\
\text { HPV 52, } 1.1\end{array}$ & $\begin{array}{l}\text { HIV Positive } \\
\text { Any HR-HPV }{ }^{\mathrm{a}}, 17.3 \\
\text { Type-specific genotypes }{ }^{a} \\
\text { HPV } 16,3.2 \\
\text { HPV } 18,2.1 \\
\text { HPV 45, } 2.7 \\
\text { HPV 51, 5.1 } \\
\text { HPV 52, } 1.3 \\
\text { HIV Negative } \\
\text { Any HR-HPV } \\
\text { Type-specific genotypes } \\
\text { HPV 16, } 1.2 \\
\text { HPV 18, } 0.7 \\
\text { HPV 45, } 0.8 \\
\text { HPV 51, } 1.2 \\
\text { HPV 52, } 1.0\end{array}$ & $\begin{array}{l}\text { HIV } \\
\text { prevalence } \\
14.0 \%\end{array}$ \\
\hline $\begin{array}{l}\text { Banura } \\
\text { et al., } \\
2010 \\
{[25]}\end{array}$ & $\begin{array}{l}\text { Teenage clinic in Kampala, } \\
380 \text { young women, median } \\
\text { age at baseline } 19 \text { years } \\
\text { (range, 12-24) }\end{array}$ & $\begin{array}{l}\text { Prospective } \\
\text { cohort }\end{array}$ & $\begin{array}{l}\mathrm{SPF}_{10} \\
\mathrm{LIPA} \\
\mathrm{PCR} \\
\text { assay }\end{array}$ & $\begin{array}{l}\text { Any HPV }{ }^{a}, 30.5 \\
\text { HR-HPV types, } 20.9 \\
\text { LR-HPV types, } 10.6 \\
\text { HPV 16-related types, } 10.8 \\
\text { HPV 18-related types, } 5.6 \\
\text { Type-specific genotypes } \\
\text { HR-HPV genotypes } \\
\text { HPV } 16,3.3 \\
\text { HPV } 18,2.0 \\
\text { HPV } 45,1.3 \\
\text { HPV 51, } 4.2 \\
\text { HPV 52, } 3.4 \\
\text { LR-HPV genotypes } \\
\text { HPV } 6,3.4 \\
\text { HPV } 11,1.9\end{array}$ & $\begin{array}{l}\text { HIV Positive } \\
\text { Any HR-HPV }{ }^{a}, 40.0 \\
\text { Type-specific genotypes } \\
\text { HPV 16, } 6.7 \\
\text { HPV 18, } 3.0 \\
\text { HIV Negative } \\
\text { Any HR-HPV }{ }^{a}, 29.7 \\
\text { Type-specific genotypes } \\
\text { HPV 16, } 3.0 \\
\text { HPV 18, } 2.1\end{array}$ & $\begin{array}{l}\text { Cumulative } \\
\text { prevalence } \\
\text { over } 27.5 \\
\text { months } \\
\text { HR-HPV } \\
\text { types, 68.2\% } \\
\text { LR-HPV } \\
\text { types, 55.0\% } \\
\text { HPV 16- } \\
\text { related } \\
\text { types, 48.6\% } \\
\text { HPV 18- } \\
\text { related } \\
\text { types, 28.2\% }\end{array}$ \\
\hline
\end{tabular}

$\mathrm{HR}^{*}=$ High Risk

$\mathrm{LR}^{* *}=$ Low Risk

${ }^{a}$ Denominator consist of all the tested women

PYs = Person Years

raising concerns of perhaps reduced immunogenicity and efficacy.

In this review, HIV positive women seemed to have multiple and more other HR-HPV genotypes than HPV $16 / 18$ consistent with a previous study where $50 \%$ of HIV positive women with HPV 16 and 18 were coinfected with other HR-HPV genotypes [38]. Consequently, it is unclear whether the current vaccines containing only HPV 16 and 18 genotypes may potentially prevent fewer cases of ICC among HIV positive women [39]. Fortunately, the current preventive vaccines have shown cross-protection against some non vaccine HRHPV genotypes and because of this, it is estimated that wide coverage ( $>70 \%$ ) could potentially prevent up to $71 \%$ of ICC [40]. Cross-protection even if limited, may be particularly important in low-resource countries like Uganda where screening programs are limited or nonexistent.

Males had a high burden of HR-HPV infections. Studies have shown that males do not perceive themselves to be susceptible to HPV and do not believe that HPV infection is a severe problem to themselves [41]. However, the role of men as vectors of HR-HPV genotypes that cause ICC has been extensively evaluated in epidemiological studies [42-44]. HPV vaccines have been found to be safe and efficacious in males [45]. However, vaccinating males does not appear to be economical when assessed from the view of ICC. Moreover, the WHO does not recommend routine male vaccination as the primary target of vaccination. Yet, the concept of herd immunity in public health would tend to suggest that vaccinating males would provide a double benefit to females in that the fewer males with HPV, the fewer females will be exposed [46].

Although HPV vaccines hold great promise to reduce ICC-associated mortality and morbidity, it remains unclear whether low-resource countries like Uganda will reap the benefits any time soon. The current high cost of the vaccines, poor health infrastructure and competing health priorities will prevent young girls getting the life saving vaccinations. Uganda's health sector remains considerably under-funded. At less than $10 \%$ of total 
Table 3 HPV prevalence and genotype distribution among invasive cervical cancer cases

\begin{tabular}{|c|c|c|c|c|c|}
\hline Author & Area, subjects & $\begin{array}{l}\text { Study } \\
\text { design }\end{array}$ & HPV test & $\begin{array}{l}\text { HPV prevalence for any and } \\
\text { specific genotypes among all } \\
\text { tested women }\end{array}$ & Comments \\
\hline $\begin{array}{l}\text { Schmauz } \\
\text { et al., } \\
1989[55]\end{array}$ & $\begin{array}{l}\text { Mulago hospital, cervical tissues from } 34 \text { cases and } 23 \\
\text { controls }\end{array}$ & $\begin{array}{l}\text { Case } \\
\text { Control }\end{array}$ & $\begin{array}{l}\text { Southern } \\
\text { Blot } \\
\text { analysis }\end{array}$ & $\begin{array}{l}\text { Cases }^{\mathrm{a}} \\
\text { HPV 16/18, 50\% } \\
\text { HPV 16, 15\% } \\
\text { HPV 18, 35\% } \\
\text { Controls } \\
\text { HPV 18, 4.4\% }\end{array}$ & $\begin{array}{l}\text { Prevalence of HIV } \\
\text { Cases, } 5.9 \% \\
\text { Controls, } 21.7 \% \\
\text { Odds of cervical } \\
\text { cancer } \\
\text { HPV } 18\left(\mathrm{OR}^{*}=\right. \\
12.0) \\
\text { HPV } 16 / 18\left(\mathrm{OR}^{*}=\right. \\
22.0)\end{array}$ \\
\hline $\begin{array}{l}\text { Odida } \\
\text { et al., } \\
2008[56]\end{array}$ & $\begin{array}{l}\text { Mulago, Pathology Department } 186 \text { paraffin embedded } \\
\text { histologically confirmed archival cervical cancer cases } \\
\text { Collected 1968-69, 1970-79, and 1980-89. }\end{array}$ & $\begin{array}{l}\text { Case } \\
\text { series }\end{array}$ & $\begin{array}{l}\mathrm{SPF}_{10} / \\
\mathrm{LIPA}\end{array}$ & $\begin{array}{l}\text { Any HPV }{ }^{a}, 61.3 \% \\
\text { Type-specific genotypes } \\
\text { HPV } 16 / 18,73.5 \% \\
\text { HPV } 16,44.7 \% \\
\text { HPV } 18,28.8 \% \\
\text { HPV } 45,9.6 \%\end{array}$ & $\begin{array}{l}\text { Types } \\
146 \text { Squamous cel } \\
\text { carcinoma } \\
35 \\
\text { adenocarcinoma } \\
3 \text { Adenosquamous } \\
2 \text { undifferentiated } \\
\text { types }\end{array}$ \\
\hline $\begin{array}{l}\text { Odida } \\
\text { et al., } \\
2010[57]\end{array}$ & $\begin{array}{l}\text { Mulago Hospital, Kampala, } 171 \text { pairs of paraffin embedded } \\
\text { and freshly frozen tissue samples collected between } \\
\text { September } 2004 \text { and December } 2006\end{array}$ & $\begin{array}{l}\text { Case } \\
\text { series }\end{array}$ & $\begin{array}{l}\mathrm{SPF}_{10} / \\
\text { LIPA }\end{array}$ & $\begin{array}{l}\text { Any HPV }{ }^{a} \text {, Frozen tissue, } 90.1 \% \\
\text { Type-specific genotypes }{ }^{a} \\
\text { HPV } 16,47.4 \% \\
\text { HPV } 18,19.5 \% \\
\text { HPV } 45,9.7 \% \\
\text { HPV } 51,1.9 \% \\
\text { HPV 52, } 1.3 \% \\
\text { Any HPV }{ }^{\text {a }} \text { Paraffin embedded } \\
\text { tissue, } 88.9 \% \\
\text { Type-specific genotypes } \\
\text { HPV } 16,47.4 \% \\
\text { HPV } 18,23.7 \% \\
\text { HPV } 45,7.9 \% \\
\text { HPV } 51,1.3 \% \\
\text { HPV 52, } 1.3 \%\end{array}$ & $\begin{array}{l}\text { Multiple HPV } \\
\text { genotypes }^{\mathrm{a}} \\
\text { Frozen tissue, 7.1\% } \text { Paraffin } \\
\text { embedded, 4.6\% } \\
\text { Single HPV } \\
\text { genotypes } \\
\text { Frozen tissue, } \\
\text { 92.9\% (95\% CI': } \\
\text { 87.6-96.4) } \\
\text { Paraffin } \\
\text { embedded, 94.7\% } \\
\text { (95\% } \mathrm{Cl}^{+}: 89.9-97.7 \text { ) }\end{array}$ \\
\hline
\end{tabular}

${ }^{a}$ Denominator consist of all the tested women

$\mathrm{Cl}^{\dagger}=$ Confidence Interval

$\mathrm{OR}^{*}=$ Odds Ratio

government expenditure in fiscal year 2010/2011, public health expenditure remains far below the Abuja target of $15 \%$ that the government of Uganda committed to in $2001[47,48]$. Furthermore, presently, there is no known public sector pricing for the HPV vaccines making it difficult to budget for and as such the details of vaccine implementation and cost coverage remain unknown. Fortunately, the Global Alliance for Vaccines and Immunization (GAVI) pledged to consider HPV vaccines in their investment strategy of 2009-2013 [49]. This may provide the much needed financial help to implement HPV immunization programs in lowresource countries where most of the ICC occur.

For prevention, HPV vaccines must be given before sexual debut to ensure that exposure to HPV has not occurred. Accordingly, the World Health Organization recommended that girls between the ages of 9-13 years should be the primary target of vaccination [50]. Given the financial impossibility of vaccinating all eligible women in the reproductive age, it is vital that primary prevention by HPV vaccination is integrated with education on risk reducing behaviors and secondary prevention programs via screening and treatment of precancerous lesions and ICC. The availability of new and inexpensive screening techniques for rapid identification of HR-HPV may help facilitate the use of HPV testing in low-resource countries [51].

\section{Conclusions}

HR-HPV infections were frequent in both females and males. HR-HPV 16 and 18 targeted by the two licensed preventive HPV vaccines are the most common genotypes found in ICC cases. However, there are a large number of individuals infected with other non vaccine HR-HPV genotypes not targeted by the currently available HPV vaccine preparations, indicating that these vaccines could be less effective and leave women at risk for ICC. Furthermore, given the high prevalence of HIV infection, HPV-associated conditions represent a major public health burden in Uganda. Thus, to further reduce this health burden, any future preventive HPV vaccine(s) should contain more HR-HPV 
Table 4 HPV prevalence in males participating in circumcision clinical trials

\begin{tabular}{|c|c|c|c|c|c|c|}
\hline Author & Area, subjects & $\begin{array}{l}\text { Study } \\
\text { design }\end{array}$ & HPV test & $\begin{array}{l}\text { HPV prevalence for } \\
H R^{*} \text {, and } L R^{* *} \\
\text { genotypes at } \\
\text { enrollment and } 24 \\
\text { month follow up in } \\
\text { intervention } \S \text { and } \\
\text { control§§ }\end{array}$ & $\begin{array}{l}\text { HPV prevalence } \\
\text { for any, } \mathrm{HR}^{*} \text { and } \\
\mathrm{LR}^{* *} \text { genotypes } \\
\text { at baseline and } \\
\text { 24-month } \\
\text { follow-up }\end{array}$ & $\begin{array}{l}\text { Risk Ratio (RR) and } \\
95 \% \mathrm{Cl} \text { for any, } \mathrm{HR}^{*}, \\
\mathrm{LR}^{* *} \text { and multiple } \\
\mathrm{HPV} \text { infections at } \\
\text { baseline and } 24- \\
\text { month follow-up }\end{array}$ \\
\hline $\begin{array}{l}\text { Tobian } \\
\text { et al., } \\
2009 \text { [58] }\end{array}$ & $\begin{array}{l}\text { Rakai district, } 307 \text { men in } \\
\text { intervention and } 233 \text { men } \\
\text { in control group aged 15-49 } \\
\text { years who had detectable } \\
\text { beta-globulin or HPV. } \\
\text { Preputal cavity sampled }\end{array}$ & $\begin{array}{l}\text { Randomized } \\
\text { controlled } \\
\text { trial }\end{array}$ & $\begin{array}{l}\text { Roche HPV linear } \\
\text { array. HR-HPV } \\
\text { genotypes 16, } \\
\text { 18,31, 33, 35, 39, 45, } \\
\text { 51,52,56, 58, 59, } \\
66 \text {, and } 68 \text { tested }\end{array}$ & $\begin{array}{l}\text { At enrollment } \\
\text { HR-HPV genotypes } \\
\text { Intervention group, }^{\mathrm{a}} \\
38.1 \% \\
\text { Control group, 37.1\% } \\
\text { At } 24 \text { months } \\
\text { HR-HPV genotypes } \\
\text { Intervention group, }^{\text {a }} \text { 18.0\% } \\
\text { Control group, 27.9\% }\end{array}$ & $\begin{array}{l}\text { Intervention } \\
\text { Any HPV } \\
\text { genotype } \\
\text { Baseline, 61.9\% } \\
\text { At } 24 \text { months, } \\
35.6 \% \\
\text { HR-HPV } \\
\text { genotypes } \\
\text { Baseline, 38.1\% } \\
\text { At } 24 \text { months, } \\
18.0 \% \\
\text { LR-HPV genotypes } \\
\text { Baseline, } 47.6 \% \\
\text { At } 24 \text { months, } \\
26.2 \% \\
\text { Control group } \\
\text { Any HPV types } \\
\text { Baseline, 62.6\% } \\
\text { At } 24 \text { months, } \\
51.2 \% \\
\text { HR-HPV } \\
\text { genotypes } \\
\text { Baseline, 37.1\% } \\
\text { At } 24 \text { months, } \\
27.9 \% \\
\text { LR-HPV genotypes }{ }^{\text {b }} \\
\text { Baseline, } 48.0 \% \\
\text { At } 24 \text { months, } \\
39.4 \%\end{array}$ & $\begin{array}{l}\text { Any HPV genotypes } \\
\text { Baseline, } 0.99 \text { (0.81- } \\
1.21) \\
\text { At } 24 \text { months, } 0.70 \\
(0.53-0.91) \\
\text { HR-HPV genotypes } \\
\text { Baseline, } 1.03(0.79- \\
1.33) \\
\text { At } 24 \text { months, } 0.65 \\
\text { (0.45-0.94) } \\
\text { LR-HPV genotypes } \\
\text { Baseline, } 0.99 \text { (0.79- } \\
1.25) \\
\text { At } 24 \text { months, } 0.66 \\
(0.49-0.91)\end{array}$ \\
\hline $\begin{array}{l}\text { Serwadda } \\
\text { et al., } \\
2010 \text { [59] }\end{array}$ & $\begin{array}{l}\text { Rakai district, uncircumcised } \\
\text { HIV positive men aged 15- } \\
49 \text { years; } 103 \text { men in } \\
\text { intervention group and } 107 \\
\text { men in control group }\end{array}$ & $\begin{array}{l}\text { Randomized } \\
\text { controlled } \\
\text { trial }\end{array}$ & $\begin{array}{l}\text { Roche HPV linear } \\
\text { array }\end{array}$ & $\begin{array}{l}\text { At enrollment } \\
\text { HR-HPV genotypes } \\
\text { Intervention group, }^{\mathrm{a}} \\
72.2 \% \\
\text { Control group, 76.6\% } \\
\text { LR-HPV genotypes } \\
\text { Intervention group, }^{\mathrm{a}} \\
85.6 \% \\
\text { Control group, 83.0\% } \\
\text { At } 24 \text { months } \\
\text { HR-HPV genotypes }{ }^{\mathrm{a}} \\
\text { Intervention; 55.3\% }_{\text {Control group; } 71.7 \%} \\
\text { LR-HPV genotypes } \\
\text { Intervention group, }^{\mathrm{a}} \\
49.4 \% \\
\text { Control group, } 77.4 \%\end{array}$ & & $\begin{array}{l}\text { At } 24 \text { months } \\
R R \text { for HR-HPV geno } \\
\text { types } 0.77(0.62-0.97) \\
\text { RR for multiple } \\
\text { genotypes } 0.53 \text { (0.33- } \\
\text { 0.83) }\end{array}$ \\
\hline $\begin{array}{l}\text { Gray et al., } \\
2010 \text { [60] }\end{array}$ & $\begin{array}{l}\text { Rakai district, uncircumcised } \\
\text { HIV negative men aged 15- } \\
49 \text { years; } 441 \text { randomized to } \\
\text { immediate circumcision } \\
\text { (intervention) and } 399 \\
\text { delayed circumcision } \\
\text { (control) }\end{array}$ & $\begin{array}{l}\text { Randomized } \\
\text { controlled } \\
\text { trial }\end{array}$ & $\begin{array}{l}\text { Roche HPV linear } \\
\text { array }\end{array}$ & $\begin{array}{l}\text { At enrollment } \\
\text { HR-HPV genotypes }{ }^{\mathrm{a}} \\
\text { Intervention arm, 39.1\% } \\
\text { Control arm, 38.6\% }\end{array}$ & & \\
\hline
\end{tabular}

$\mathrm{HR}^{*}=$ High Risk

$\mathrm{LR}^{* *}=$ Low Risk

$\mathrm{Cl}^{\dagger}=$ Confidence Intervals

a Denominator include all women tested

${ }^{b}$ Denominator include only HPV positive women

${ }^{5}$ Intervention refers to circumcision arm of the study

${ }^{\S}$ Control refers to non circumcision arm of the study 
Table 5 HPV incidence in males participating in circumcision clinical trials

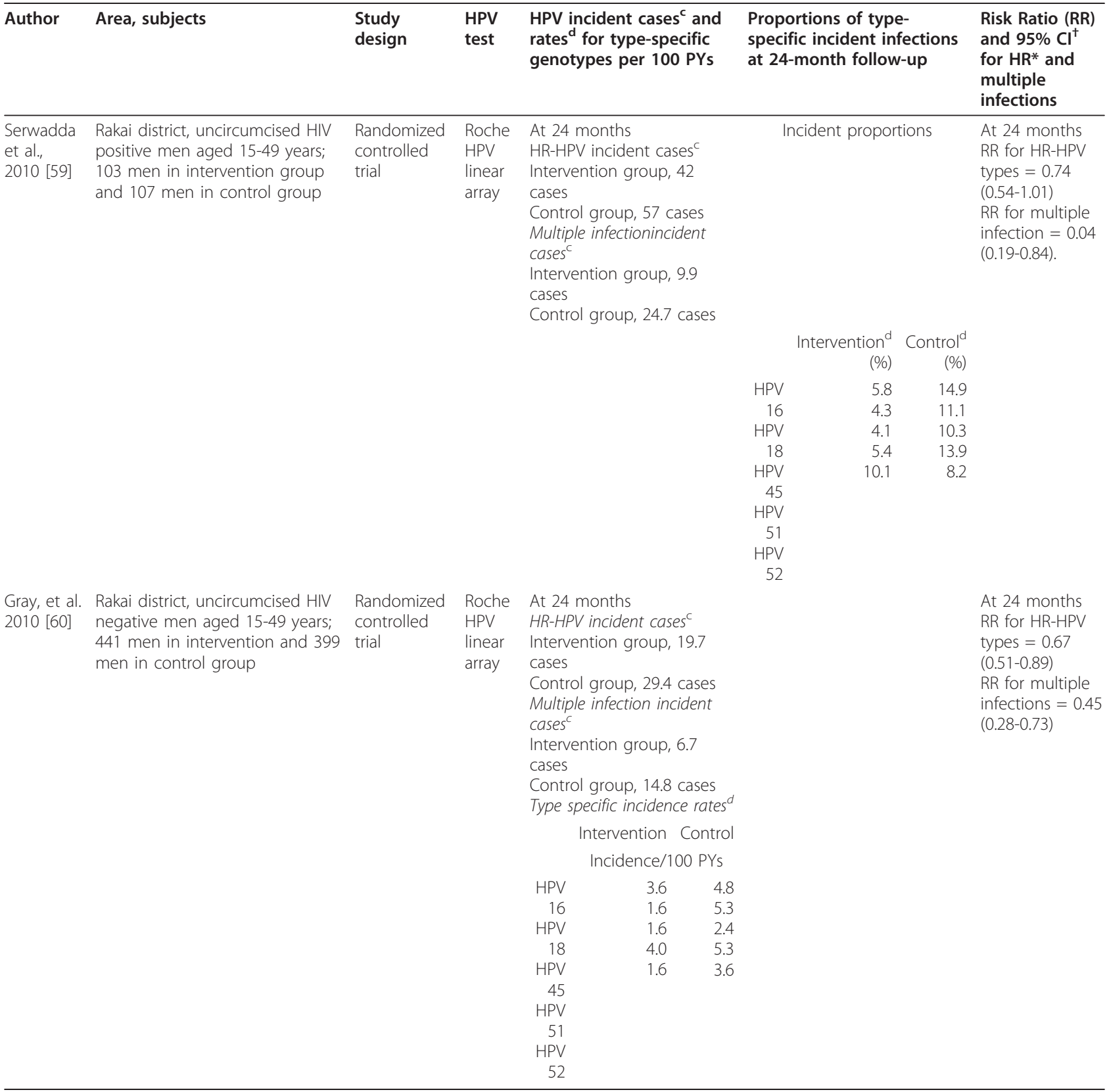

$\mathrm{HR}^{*}=$ High Risk

$\mathrm{Cl}^{\dagger}=$ Confidence Intervals

PYs $=$ Person Years

C Denominator include all women tested

${ }^{d}$ Denominator include women with HR-HPV 
Table 6 HPV Seroprevalence among women with and without invasive cervical cancer

\begin{tabular}{|c|c|c|c|c|c|c|}
\hline Author & Area, subjects & $\begin{array}{l}\text { Study } \\
\text { design }\end{array}$ & HPV test & $\begin{array}{l}\text { HPV } \\
\text { seroprevalence } \\
\text { for any and } \\
\text { type specific } \\
\text { genotypes }\end{array}$ & $\begin{array}{l}\text { HPV seroprevalence of } \\
\text { any and type specific } \\
\text { genotypes among HIV } \\
\text { positive women }\end{array}$ & Comments \\
\hline $\begin{array}{l}\text { Newton, } \\
\text { et al., } \\
\text { 2004) } \\
{[27]}\end{array}$ & $\begin{array}{l}\text { Mulago hospital, } 191 \text { cervical cancer } \\
\text { cases and } 336 \text { controls; } 15 \text { years and } \\
\text { older with a new diagnosis of cancer } \\
\text { from the wards or outpatient clinics } \\
\text { between } 1994 \text { and } 1998 .\end{array}$ & $\begin{array}{l}\text { Case } \\
\text { Control }\end{array}$ & $\begin{array}{l}\text { HPV L1 } \\
\text { VLP/ELISA }\end{array}$ & $\begin{array}{l}\text { Cases } \\
\text { Type-specific } \\
\text { genotypes } \\
\text { HPV 16, 27\% } \\
\text { HPV 18, 7\% } \\
\text { HPV 45, 9\% } \\
\text { Controls } \\
\text { Any of } 3 \text { HPV } \\
\text { types, } 17 \% \\
\text { Type-specific } \\
\text { genotypes } \\
\text { HPV 16, 11\% } \\
\text { HPV 18, 5\% } \\
\text { HPV 45, 6\% }\end{array}$ & $\begin{array}{l}\text { HIV Positive Controls } \\
\text { Any of } 3 \text { HPV types, } \\
\text { 22\% Type-specific } \\
\text { genotypes } \\
\text { HPV } 16,16 \% \\
\text { HPV } 18,7 \% \\
\text { HPV } 45,8 \%\end{array}$ & $\begin{array}{l}\text { Antibodies against HPV } \\
16 \text { were significantly } \\
\text { associated with cervical } \\
\text { cancer } \mathrm{OR}^{*}=2.0(1.2-3.1) \\
\text { The risk increased with } \\
\text { increasing anti-HPV } 16 \\
\text { antibody titre ( } P_{\text {trend }}= \\
0.01)\end{array}$ \\
\hline $\begin{array}{l}\text { Namujju, } \\
\text { et al., } \\
2010[26]\end{array}$ & $\begin{array}{l}\text { Naguru and Nsambya Health Centers in } \\
\text { Kampala, 2,053 women seeking } \\
\text { antenatal services; mean age } 23 \text { years } \\
\text { (range, 14-48) }\end{array}$ & $\begin{array}{l}\text { Cross- } \\
\text { sectional }\end{array}$ & $\begin{array}{l}\text { Serology/ } \\
\text { VLP ELISA } \\
(6,11 \\
16,18,31 \\
33,45)\end{array}$ & $\begin{array}{l}\text { Any of the } 7 \\
\text { selected HPV } \\
\text { types }^{a}, 57 \%\end{array}$ & & HIV prevalence ${ }^{a}, 7.0 \%$ \\
\hline
\end{tabular}

${ }^{\mathrm{a} D e n o m i n a t o r}$ consist of all tested women

$\mathrm{OR}^{*}=$ Odds Ratio

genotypes than only two targeted by the current vaccines.

\section{Author details}

${ }^{1}$ Child Health and Development Centre, Makerere University College of Health Sciences, P. O. Box 6717, Kampala, Uganda. ²Department of Obstetrics and Gynecology, Makerere University College of Health Sciences, P.O. Box 7072, Kampala Uganda. ${ }^{3}$ Uganda Virus Research Institute, P.O. Box 49, Entebbe, Uganda. ${ }^{4}$ National Institute for Health and Welfare, Oulu, Finland. ${ }^{5}$ Department of Community Health Ministry of Health, P.O. Box 7272, Kampala, Uganda. 'SChool of Public Health, Makerere University College of Health Sciences, P.O. Box 7072, Kampala, Uganda.

\section{Authors' contributions}

CB conceived the study, searched the literature, drafted the manuscript and produced the final tables. MMF, KRA, NBP, MKA \& WMF made substantial contributions to the manuscript and contributed to data interpretation. All authors read and approved the final manuscript.

\section{Competing interests}

The authors declare that they have no competing interests.

Received: 7 February 2011 Accepted: 12 July 2011

Published: 12 July 2011

\section{References}

1. Ferlay J, Bray F, Parkin MD: GLOBOCAN 2002: Cancer incidence, mortality and prevalence worldwide. IARC cancer Base. No. 5 version 2.0 IARC, Lyon; 2004.

2. Walboomers JM, Jacobs MV, Manos MM, Bosch FX, Kummer JA, Shah KV, Snijders PJ, Peto J, Meijer CJ, Muñoz N: Human papillomavirus is a necessary cause of invasive cervical cancer worldwide. J Pathol 1999, 189(1):12-9.

3. Koutsky L: Epidemiology of genital human papillomavirus infection. Am J Med 1997, 102(5A):3-8.

4. Moscicki AB, Schiffman M, Kjaer S, Villa LL: Chapter 5: Updating the natural history of HPV and anogenital cancer. Vaccine 2006, 24(Suppl 3):S3/42-51.

5. Nielson CM, Flores R, Harris RB, Abrahamsen M, Papenfuss MR, Dunne EF, Markowitz LE, Giuliano AR: Human Papillomavirus Prevalence and Type
Distribution in Male Anogenital Sites and Semen. Cancer Epidemiol Biomarkers Prev 2007, 16(6):1107-14[http://cebp.aacrjournals.org/content/16/ 6/1107.abstract - target-2].

6. IARC:[http://globocan.iarc.fr]

7. Muñoz N, Bosch FX, de Sanjosé S, Herrero R, Castellsagué X, Shah KV, Snijders PJF, Meijer CJLM, the International Agency for Research on Cancer Multicenter Cervical Cancer Study Group: Epidemiologic classification of human papillomavirus types associated with cervical cancer. N Eng J Med 2003, 348:518-27.

8. Lacey CJN, Lowndes CM, Shah KV: Chapter 4: Burden and management of non-cancerous HPV-related conditions: HPV-6/11 disease. Vaccine 2006, 24S3:S3/35-S3/41.

9. The WHO/ICO Information Centre on HPV and Cervical Cancer. [http:// www.who.int/hpvcentre/en/]

10. Parkin MD, Bray F, Ferlay J, Pisani P: Global Cancer Statistics, 2002. CA: A Cancer J Clinicians 2005, 55(2):74-108.

11. PATH: Cervical cancer vaccine. Cervical cancer.[http://www.path.org/ projects/cervical_cancer_vaccine.php].

12. Mariani L: HPV-vaccine and screening programs: the new era of global prevention. J Preve Med Hyg 2009, 50:90-5.

13. Cope U, Hildesheim A, Schiffman MH, Manos MM, Lorincz AT, Burk RD, Glass AG, Greer C, Buckland J, Helgesen K, Scott DR, Sherman ME, Kurman RJ, Liaw KL: Comparison of the hybrid capture tube test and PCR for detection of human papillomavirus DNA in cervical specimens. J Clin Microbiol 1997, 35(9):2262-5.

14. Quint W, Pagliusi SR, Lelie N, de Villiers EM, Wheeler CM, the World Health Organization Human Papillomavirus DNA International Collaborative Study Group: Results of the First World Health Organization International Collaborative Study of Detection of Human Papillomavirus DNA. J Clin Microbiol 2006, 44(2):571-9.

15. Chan PK, Cheung TH, Tam AO, Lo KW, Yim SF, Yu MM, To KF, Wong YF, Cheung $J$, Chan DP, Hui M, Ip M: Biases in human papillomavirus genotype prevalence assessment associated with commonly used consensus primers. Int J Cancer 2006, 118(1):243-5.

16. Banura C, Franceschi S, Doorn LJ, Arslan A, Wabwire-Mangen F, Mbidde EK, Quint W, Weiderpass E: Infection with human papillomavirus and HIV among young women in Kampala, Uganda. J Infect Dis 2008, 197(4):555-62.

17. Blossom DB, Beigi RH, Farrell JJ, Mackay W, Qadadri B, Brown DR, Rwambuya S, Walker CJ, Kambugu FS, Abdul-Karim FW, Whalen CC, Salata RA: Human papillomavirus genotypes associated with cervical 
cytologic abnormalities and HIV infection in Ugandan women. J Med Virol 2007, 79(6):758-65.

18. Banura C, Franceschi S, van Doorn LJ, Arslan A, Kleter B, WabwireMangen F, Mbidde EK, Quint W, Weiderpass E: Prevalence, incidence and clearance of human papillomavirus infection among young primiparous pregnant women in Kampala, Uganda. Int J Cancer 2008, 123(9):2180-7.

19. Taube JM, Kamira B, Motevalli M, Nakabiito C, Lukande R, Kelly DP, Erozan YS, Gravitt PE, Buresh ME, Mmiro F, Bagenda D, Guay LA, Jackson JB: Human papillomavirus prevalence and cytopathology correlation in young Ugandan women using a low-cost liquid-based Pap preparation. Diagn Cytopathol 2010, 38(8):555-63.

20. Safaeian M, Kiddugavu M, Gravitt PE, Gange SJ, Ssekasanvu J, Murokora D, Sklar M, Serwadda D, Wawer MJ, Shah KV, Gray R: Prevalence and risk factors for carcinogenic human papillomavirus infections in rural Rakai, Uganda. Sex Transm Infect 2008, 84:306-311[http://sti.bmj.com/content/84/ 4/306.abstract-aff-1],

21. Asiimwe S, Whalen CC, Tisch DJ, Tumwesigye E, Sethi AK: Prevalence and predictors of high-risk human papillomavirus infection in a populationbased sample of women in rural Uganda. Int J STD AIDS 2008, 19(9):605-10.

22. Clifford G, Smith JS, Aguado T, Franceschi S: Comparison of HPV type distribution in high-grade cervical lesions and cervical cancer: a metaanalysis. British J Cancer 2003, 89:101-5.

23. Smith JS, Melendy A, Rana RK, Pimenta JM: Age-specific prevalence of infection with human papillomavirus in females: a global review. J Adolesc Health 2008, 43(Suppl. 4):S5-25.

24. Safaeian M, Kiddugavu M, Gravitt PE, Gange SJ, Ssekasanvu J, Murokora D, Sklar M, Serwadda D, Wawer MJ, Shah KV, Gray R: Determinants of Incidence and Clearance of High-Risk Human Papillomavirus Infections in Rural Rakai, Uganda. Cancer Epidemiol Biomarkers Prev 2008, 17(6):1300-7 [http://cebp.aacrjournals.org/content/17/6/1300.abstract-target-4].

25. Banura C, Sandin S, van Doorn L, Quint W, Kleter B, Wabwire-Mangen F, Mbidde EK, Weiderpass E: Type-specific incidence, clearance and predictors of cervical human papillomavirus infections (HPV) among young women: a prospective study in Uganda. Infectious Agents and Cancer 2010, 5:7.

26. Namujju PB, Surcel HM, Kirnbauer R, Kaasila M, Banura C, Byaruhanga R, Muwanga M, Mbidde EK, Koskela P, Lehtinen M: Risk of being seropositive for multiple human papillomavirus types among Finnish and Ugandan women. Scand J Infect Dis 2010, 42(6-7):522-6.

27. Newton R, Bousarghin L, Ziegler J, Casabonne D, Beral V, Mbidde E, Carpenter L, Parkin DM, Wabinga H, Mbulaiteye S, Jaffe H, Touzé A, Coursaget P: Uganda Kaposi's Sarcoma Study Group. Human papillomaviruses and cancer in Uganda. Eur J Cancer Prev 2004, 13(2):113-8.

28. Clifford G, Franceschi S: HPV in Sub Saharan Africa. Papillomavirus Report 2005, 16:322-6.

29. de Sanjosé S, Diaz M, Castellsagué X, Clifford G, Bruni L, Muñoz N, Bosch FX: Worldwide prevalence and genotype distribution of cervical human papillomavirus DNA in women with normal cytology: a metaanalysis. Lancet Infect Dis 2007, 7(7):453-9.

30. Frazer IH: Chapter 8: HPV Vaccines. Int J Gynecol \& Obst 2006, 94(Suppl 1): S81-S88.

31. Koutsky LA, Harper DM: Chapter 13: Current findings from prophylactic HPV vaccine trials. Vaccine 2006, 24(Suppl 3):S3/114-S3/121.

32. Hildesheim A, Wang SS: Host and viral genetics and risk of cervical cancer: a review. Virus Res 2002, 89:229-40.

33. Moscicki AB, Schiffman M, Kjaer S, Villa LL: Chapter 5: Updating the natural history of HPV and anogenital cancer. Vaccine 2006, 24(Suppl 3):S3/42-51.

34. UNAIDS: UNGASS Country Progress Report.[http://www.unaids.org/en/ dataanalysis/monitoringcountryprogress/ 2010progressreportssubmittedbycountries/ uganda_2010_country_progress_report_en.pdf].

35. De Vuyst H, Lillo F, Broutet N, Smith JS: HIV, human papillomavirus, and cervical neoplasia and cancer in the era of highly active antiretroviral therapy. Eur J Cancer Prev 2008, 17(6):545-54.

36. Parham GP, Mwanahamuntu MH, Sahasrabuddhe W, Westfall AO, King KE, Chibwesha C, Pfaendler KS, Mkumba G, Mudenda V, Kapambwe S, Vermund SH, Hicks ML, Stringer JSA, Chi BH: Implementation of cervical cancer prevention services for HIV-infected women in Zambia: measuring program effectiveness. HIV Ther 2010, 4(6):713-22.
37. Levin MJ, Moscicki AB, Song LY, Fenton T, Meyer WA, Read JS, Handelsman EL, Nowak B, Sattler CA, Saah A, Radley DR, Esser MT, Weinberg A, the IMPAACT P1047 Protocol Team: Safety and Immunogenicity of a Quadrivalent Human Papillomavirus (Types 6, 11, 16, and 18) Vaccine in HIV-Infected Children 7 to 12 Years Old. J Acquir Immune Defic Syndr 2010, 55(2):197-204.

38. De Vuyst H, Gichangi P, Estambale B, Njuguna E, Franceschi S, Temmerman M: Human papillomavirus types in women with invasive cervical carcinoma by HIV status in Kenya. Int J Cancer 2008, 122(1):244-6.

39. Louie KS, de Sanjosé S, Mayaud P: Epidemiology and prevention of human papillomavirus and cervical cancer in sub-Saharan Africa: a comprehensive review. Trop Med Int Health 2009, 14(10):1287-302.

40. Paavonen J, Naud P, Salmeron J, Wheeler CM, Chow SN, Apter D, Kitchner H, Castellsagué X, Teixeira JC, Skinner SK, Hedrick J, Jaisamrarn U, Limson G, Garland S, Szarewski A, Romanowski B, Aoki FY, Schwarz TF, Poppe WAJ, Bosch FX, Jenkins D, Hardt K, Zahaf T, Descamps D, Struyf F, Lehtinen M, Dubin G, the HPV PATRICIA STUDY GROUP: Efficacy of human papillomavirus (HPV)-16/18AS04-adjuvanted vaccine against cervical infection and precancer caused by oncogenic HPV types (PATRICIA):final analysis of a double-blind, randomised study in young girls. Lancet 2009, 374(9686):301-14.

41. McPartland TS, Weaver BA, Lee SK, Koutsky LA: Men's perceptions and knowledge of human papillomavirus (HPV) infection and cervical cancer. J Am Coll Health 2005, 53(5):225-30.

42. Skegg DC, Corwin PA, Paul C, Doll R: Importance of the male factor in cancer of the cervix. Lancet 1982, 2(8298):581-3.

43. Bosch FX, Castellsague X, Munoz N, de Sanjose S, Ghaffari AM, Gonzlez LC, Gili M, Izarzugaza I, Viladiu P, Navarro C, Vergara A, Ascunce N, Guerrero E, Keerti V, Shah KV: Male Sexual Behavior and Human Papillomavirus DNA: Key Risk Factors for Cervical Cancer in Spain. J Natl Cancer Inst 1996, 88:1060-7.

44. Castellsagué X, Bosch FX, Muñoz N, Meijer CJLM, Shah KV, de Sanjosé S, Eluf-Neto J, Ngelangel CA, Chichareon S, Smith JS, Herrero R, Moreno V, Franceschi S, the International Agency for Research on Cancer Multicenter Cervical Cancer Study Group: Male Circumcision, Penile Human Papillomavirus Infection, and Cervical Cancer in Female Partners. N Engl J Med 2002, 346:1105-1112.

45. Giuliano AR, Palefsky JM, Goldstone S, Moreira ED, Penny ME, Aranda C, Vardas E, Moi H, Jessen H, Hillman R, Chang YH, Ferris D, Rouleau D, Bryan J, Marshall JB, Vuocolo S, Barr E, Radley D, Haupt RM, Guris D: Efficacy of Quadrivalent HPV Vaccine against HPV Infection and Disease in Males. N Engl I Med 2011, 364:401-411.

46. Garnett GP: Role of herd immunity in determining the effect of vaccines against sexually transmitted disease. J Infect Dis 2005, 191(Suppl 1): S97-S106.

47. GOU: Approved budget estimates FY 2010/2011 Volume 1: Central Government Votes. Kampala 2010.

48. OAU: Abuja declaration on HIV/AIDS, Tuberculosis and other related infectious diseases. Abuja 2001.

49. GAVI Alliance: Vaccine Investiment Strategy. 2008.

50. WHO: Position Paper: Human papillomavirus vaccines.[http://www.who. int/wer/2009/wer8415.pdf].

51. Lorincz AT: HPV testing by hybrid capture. Emerging Issues of HPV Infections: From Science to Practice.Edited by: Monsonego J. Basel: Karger; 2006:54-62.

52. Serwadda D, Wawer MJ, Shah KV, Sewankambo NK, Daniel R, Li C, Lorincz A, Meehan MP, Wabwire-Mangen F, Gray RH: Use of a Hybrid Capture Assay of self-collected vaginal swabs in rural Uganda for detection of human papillomavirus. J Infect Dis 1999, 180(4):1316-9.

53. Safaeian M, Kiddugave M, Gravitt PE, Ssekasanvu J, Murokora D, Sklar M, Serwadda D, Wawer MJ, Shah KV, Gray R: Comparability of self-collected vaginal swabs and physcian collected cervical swabs for detection of human papillomavirus infections in Rakai, Uganda. Sex Transm Dis 2007, 34(7):429-36.

54. Buonaguro FM, Tornesello ML, Salatiello I, Okong P, Buonaguro L, BethGiraldo E, Biryahwaho B, Sempala SD, Giraldo G: The Uganda study on HPV variants and genital cancers. J Clin Virol 2000, 19(1-2):31-41.

55. Schmauz R, Okong P, de Villiers EM, Dennin R, Brade L, Lwanga SK, Owor R: Multiple infections in cases of cervical cancer from a high-incidence area in tropic Africa. Int J Cancer 1989, 43(5):805-9. 
56. Odida M, de Sanjosé S, Quint W, Bosch FX, Klaustermeier J, Weiderpass E: Human Papillomavirus type distribution in invasive cervical cancer in Uganda. BMC Infect Dis 2008, 8:85.

57. Odida M, de Sanjosé S, Sandin S, Quiros B, Alemany L, Lloveras B, Quint W, Kleter B, Alejo M, van Doorn L, Weiderpass E: Comparison of human papillomavirus detection between freshly frozen tissue and paraffin embedded tissue of invasive cervical cancer. Infect Agent Cancer 2010, 5:15.

58. Tobian AR, Serwadda D, Quinn TC, Kigozi G, Gravitt PE, Laeyendecker O, Charvat B, Ssempijja V, Riedesel M, Oliver AE, Nowak RG, Moulton LH, Chen MZ, Reynolds SJ, Wawer MJ, Gray RH: Male Circumcision for the Prevention of HSV-2 and HPV Infections and Syphilis. N Engl J Med 2009, 360:1298-1309.

59. Serwadda D, Wawer MJ, Makumbi F, Kong X, Kigozi G, Gravitt P, Watya S, Nalugoda F, Ssempijja V, Tobian AAR, Kiwanuka N, Moulton LH, Sewankambo NK, Reynolds SJ, Quinn TC, Oliver ME, Iga B, Laeyendecker O, Gray RH: Circumcision of HIV-infected men: Effects on High Risk Human Papillomavirus Infections in a Randomized Trial in Rakai, Uganda. J Infect Dis 2010, 201(10): 1463-9.

60. Gray RH, Serwadda D, Kong X, Makumbi F, Kigozi G, Gravitt PE, Watya S, Nalugoda F, Ssempijja V, Tobian AA, Kiwanuka N, Moulton LH, Sewankambo NK, Reynolds SJ, Quinn TC, Iga B, Laeyendecker O, Oliver AE, Wawer MJ: Male circumcision decreases acquisition and increases clearance of high-risk human papillomavirus in HIV-negative men: a randomized trial in Rakai, Uganda. J Infect Dis 2010, 201(10):1455-62.

doi:10.1186/1750-9378-6-11

Cite this article as: Banura et al: Epidemiology of HPV genotypes in Uganda and the role of the current preventive vaccines: A systematic review. Infectious Agents and Cancer 2011 6:11.

\section{Submit your next manuscript to BioMed Central and take full advantage of:}

- Convenient online submission

- Thorough peer review

- No space constraints or color figure charges

- Immediate publication on acceptance

- Inclusion in PubMed, CAS, Scopus and Google Scholar

- Research which is freely available for redistribution

Submit your manuscript at www.biomedcentral.com/submit 